\title{
Assessing the population impact of low rates of breast feeding on asthma, coeliac disease and obesity: the use of a new statistical method
}

\author{
Anthony K Akobeng, Richard F Heller
}

Arch Dis Child 2007;92:483-485. doi: 10.1136/adc.2006.097667

See end of article for authors' affiliations

......................

Correspondence to: Dr A K Akobeng, Department of Paediatric Gastroenterology, Centra Manchester and Manchester Children's University Hospitals, Booth Hall Children's Hospital, Charlestown Road, Blackley, Manchester M9 7AA, UK; tony.akobeng@cmmc.nhs.uk

Accepted 10 July 2006

Published Online First 13 July 2006
Background: Lack of breast feeding has been reported to be associated with a number of chronic childhood disorders.

Aim: To use a recently described measure, the population impact number of eliminating a risk factor over a time period (PIN-ER-t), to quantify the burden of low rates of breast feeding in a UK population of babies born in 2002 with regard to asthma, coeliac disease and obesity.

Methods: We performed literature searches for systematic reviews with meta-analyses that had investigated the association between breast feeding and asthma, coeliac disease and obesity. Based on these data, and published data on the prevalence of breast feeding and the prevalence of the disorders, we calculated PINER- $t$ and estimated the number of cases of each disorder which could be prevented by eliminating "no breast feeding" as a risk factor.

Results: In the population of the 596122 babies born in England and Wales in 2002, the number of cases of asthma, coeliac disease and obesity that could be prevented over 7-9 years if "no breast feeding" as a risk factor was eliminated were 33100 (95\% Cl 17710 to 47543 ), 2655 (95\% Cl 1937 to 3343) and 13639 (95\% Cl 7838 to 19308), respectively.

Conclusions: The population burden of low breast feeding rates is high with regard to these chronic disorders. The use of PIN-ER-tallows the population burden of low breast feeding rates to be quantified and communicated in a way that will make it easier for both the general public and decision makers to understand.
A lthough the many nutritional, growth, and immunological benefits of breast feeding are well documented, ${ }^{1}$ rates of breast feeding remain low in many countries. ${ }^{2}$ Recent epidemiological studies suggest that breast feeding may also reduce the risk of certain chronic childhood disorders. ${ }^{3}$

Traditionally, the results of studies investigating the association between a risk factor and a disorder are usually reported in measures of relative risk or odds ratio, but these measures do not give a clear indication of the burden of the risk factor on the population, as they do not take account of the prevalence of the risk factor in the population. Other epidemiological measures of relative risk that do take this into account, such as the population attributable risk (PAR), are difficult to conceptualise and remember and may be incomprehensible to non-epidemiologists. ${ }^{4}$ None of these measures indicates in absolute terms the benefits that the population might gain if breast feeding rates are improved.

Heller et al have recently described a new measure, the "population impact number of eliminating a risk factor over a time period (PIN-ER- $t$ )" which is defined as the potential number of disease events prevented in a population over the next $t$ years by eliminating a risk factor. ${ }^{4}$ This statistic is easily calculated and allows numbers to be communicated in a more friendly way to show the impact of risk factors for disease in a population to both decision makers and the general public.

In this article, we will explain PIN-ER- $t$ and apply it to three childhood chronic disorders which have been reported to be associated with no breast feeding at specified times, in order to quantify the population burden of low breast feeding rates on a United Kingdom (UK) population.

\section{METHODS}

We searched Medline (PubMed) (1966 to January 2006) for systematic reviews with meta-analyses that had reported odds ratio (OR) or relative risk (RR) and 95\% confidence intervals (95\% CI) for the association between breast feeding and three chronic disorders (asthma, coeliac disease and obesity). We performed separate searches for each of the three conditions by combining the term "breast-feeding OR breast feeding" with the name of the particular condition and its synonyms. Where more than one meta-analysis was found on a particular condition, the result of the most recent study was used. The main result of each meta-analysis was used in the calculation of PIN-ER- $t$ (see below). Where ORs were used to report results of meta-analyses, we assumed that OR approximates to RR. ${ }^{5}$

\section{Population}

We applied PIN-ER- $t$ to the 596122 infants born in England and Wales in 2002. ${ }^{6}$ Prevalence of breast feeding at different times of infancy was estimated from a recent UK survey. ${ }^{7}$ The UK childhood incidence of each of the chronic disorders was estimated from published data.

\section{Calculating PIN-ER-t}

For a dichotomous variable such as breast feeding or no breast feeding, PIN-ER- $t$ is calculated as the population size multiplied by the risk of an event in the next $t$ years, multiplied by the population attributable risk (PAR): ${ }^{4}$

PIN-ER- $t=\mathrm{N} \times \mathrm{Ip} \times \mathrm{PAR}$

where $\mathrm{PAR}=(\mathrm{Pe}(\mathrm{RR}-1)) /(\mathrm{l}+\mathrm{Pe}(\mathrm{RR}-\mathrm{l})), \mathrm{N}$ is population size, Ip is incidence of the outcome in the whole population over $t$ years, Pe is proportion of the population with the risk factor, and $\mathrm{RR}$ is relative risk of an outcome event if the risk factor is present.

Abbreviations: $\mathrm{Cl}$, confidence interval; $\mathrm{OR}$, odds ratio; PAR, population attributable risk; PIN-ER-t, population impact number of eliminating a risk factor over a time period 
PIN-ER- $t$ and 95\% CI were calculated using the online PINER-t calculator, version 3 (http://www.phsim.man.ac.uk/pinert/ Default.aspx).

PIN-ER- $t$ was used to estimate the number of cases of asthma, coeliac disease and obesity that could be prevented if "no breast feeding" as a risk factor was eliminated at specified time periods. Since it would be impractical to expect all mothers to breast feed (ie, to completely eliminate "no breast feeding" as a risk factor), we also calculated the number of cases of the disorders which could be prevented if current breast feeding rates are improved to higher prevalence levels.

\section{RESULTS}

\section{Asthma}

Gdalevich et al conducted a systematic review of studies that evaluated the association between breast feeding during the first 3 months after birth and childhood asthma. ${ }^{8}$ Twelve studies from Europe, North America and Australia (involving 8183 participants) were included in meta-analyses. They found that breast feeding during the first 3 months after birth was associated with a reduced risk of asthma during childhood (OR $0.70 ; 95 \%$ CI 0.60 to 0.81 ). Assuming that OR approximates to $\mathrm{RR}$, the RR of asthma due to "no breast feeding" throughout the first 3 months of life is $1 / 0.7=1.43$. Based on the UK national survey, ${ }^{7}$ we estimated that about $30 \%$ of the babies in England and Wales were breast feeding throughout the first 3 months of life, which means that $70 \%$ of the babies were not breast fed (Pe) throughout this time period.

Using these RR and Pe figures, and assuming that the incidence of asthma over 8 years is the same as the parentreported asthma prevalence of $24 \%$ in $7-9$ year old children in

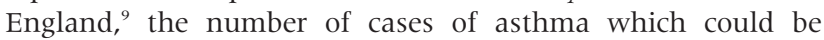
prevented over 8 years if "no breast feeding during the first 3 months of life" as a risk factor was eliminated in this population (PIN-ER-8 years) is 33100 (95\% CI 17710 to 47 543). Table 1 shows the number of cases of asthma that could be prevented over 8 years if the current breast feeding prevalence was to increase to higher levels.

\section{Coeliac disease}

In a recent meta-analysis of four European studies (involving 1969 participants), we found that the risk of coeliac disease is significantly reduced in infants being breast fed at the time of gluten introduction compared to infants who were not being breast fed at this time (OR 0.48 ; $95 \%$ CI 0.40 to 0.59 ). ${ }^{10}$ Assuming that OR approximates to relative risk (RR), the RR of coeliac disease due to "no breast feeding at the time of gluten introduction" is $1 / 0.48=2.1$. We assumed that the incidence of coeliac disease in the children over 7 years of age would be similar to the reported prevalence of 1\% in English 7 year olds. ${ }^{11}$

Table 1 Number of cases of asthma that could be prevented over 8 years at different prevalence levels of "breast feeding throughout the first 3 months of life" (compared to the baseline prevalence of $30 \%$ )

\begin{tabular}{lll}
\hline $\begin{array}{l}\text { Prevalence of } \\
\text { breast feeding } \\
(\%)^{*}\end{array}$ & $\begin{array}{l}\text { Prevalence of } \\
\text { no breast feeding } \\
(\%)^{*}\end{array}$ & $\begin{array}{l}\text { Cases of asthma } \\
\text { prevented }(95 \% \mathrm{Cl})\end{array}$ \\
\hline 30 & 70 & - \\
40 & 60 & $3759(2139$ to 5049$)$ \\
54 & 46 & $9475(5569$ to 12409$)$ \\
60 & 40 & $12104(6943$ to 15958$)$ \\
70 & 30 & $16753(9531$ to 22496$)$ \\
100 & 0 & $33100(17710$ to 47543$)$ \\
\hline
\end{tabular}

*Refers to breast feeding or no breast feeding throughout the first 3 months of life.
The prevalence of breast feeding at the time of gluten introduction was estimated to be about $27 \% .^{7}$ This means that $73 \%$ of babies are not being breast fed at the time of the introduction of solid food (Pe).

Assuming that the time of introduction of solid food is approximately equal to the time of introduction of gluten, and using these RR and Pe figures, the number of cases of coeliac disease which could be prevented over 7 years if "no breast feeding at the time of gluten introduction" as a risk factor was eliminated in this population (PIN-ER-7 years) is 2655 (95\% CI 1937 to 3343). Table 2 shows the number of cases of coeliac disease that could be prevented over 7 years if the current breast feeding prevalence was to increase to higher levels.

\section{Obesity}

In a meta-analysis of 17 studies from Europe, North America and Australia, Harder et al demonstrated that breast feeding for up to 3 months of age is associated with a reduced risk of obesity in later life (OR 0.81; 95\% CI 0.74-0.88). ${ }^{12}$ Assuming that OR approximates to RR, the RR of obesity due to lack of breast feeding for up to 3 months is estimated to be $1 /$ $0.81=1.23$. We estimated that only about $30 \%$ of babies in England and Wales breast feed for up to 3 months, ${ }^{7}$ which means that $70 \%$ of the babies were not breast fed for up to 3 months (Pe). Using these RR and Pe figures, and assuming the incidence of obesity in the babies over 9 years would be the same as the 2003 prevalence of obesity (defined as a body mass index greater than the 95th centile) in English children aged 810 years which was $16.5 \%,{ }^{13}$ PIN-ER-9 years or the number of cases of obesity which could be prevented over 9 years if "no breast feeding during the first 3 months of life" as a risk factor was eliminated in this population was calculated to be 13639 (95\% CI 7838 to 19308 ). Table 3 shows the number of cases of obesity that could be prevented over 9 years if the current breast feeding prevalence was to increase to higher levels.

\section{DISCUSSION}

Communicating public health risk to the general public and decision makers can be a challenging task. Many of the epidemiological measures used to report risk such as RR and OR may indicate how strongly exposure and disease are associated, but they do not indicate directly the benefits that could be gained by the population if the exposure is modified or eliminated. ${ }^{14}$ PIN-ER-t, a recently described measure, allows the population burden of a risk factor to be quantified and expressed in a way that both healthcare professionals and the general public will find easier to conceptualise.

In this paper, we have calculated PIN-ER- $t$ for three chronic childhood conditions in order to estimate the population burden of low rates of breast feeding on babies born in the

Table 2 Number of cases of coeliac disease that could be prevented over 7 years at different prevalence levels of "breast feeding at the time of gluten introduction" (compared to the baseline prevalence of $27 \%$ )

\begin{tabular}{lll}
\hline $\begin{array}{l}\text { Prevalence of } \\
\text { breast feeding } \\
(\%)^{*}\end{array}$ & $\begin{array}{l}\text { Prevalence of } \\
\text { no breast feeding } \\
(\%)^{*}\end{array}$ & $\begin{array}{l}\text { Cases of coeliac disease } \\
\text { prevented }(95 \% \mathrm{CI})\end{array}$ \\
\hline 27 & 73 & - \\
40 & 60 & $284(220$ to 344$)$ \\
54 & 46 & $652(513$ to 770$)$ \\
60 & 40 & $833(653$ to 983$)$ \\
70 & 30 & $1175(913$ to 1394$)$ \\
100 & 0 & $2655(1937$ to 3343$)$ \\
\hline
\end{tabular}

*Refers to breast feeding or no breast feeding at the time of gluten introduction. 
Table 3 Number of cases of obesity (defined as a body mass index greater than the 95th centile) that could be prevented over 9 years at different prevalence levels of "breast feeding for up to 3 months" (compared to the baseline prevalence of $30 \%$ )

\begin{tabular}{lll}
\hline $\begin{array}{l}\text { Prevalence of } \\
\text { breast feeding } \\
(\%)^{*}\end{array}$ & $\begin{array}{l}\text { Prevalence of } \\
\text { no breast feeding } \\
(\%)^{*}\end{array}$ & $\begin{array}{l}\text { Cases of obesity } \\
\text { prevented }(95 \% \mathrm{CI})\end{array}$ \\
\hline 30 & 70 & - \\
40 & 60 & $1712(1039$ to 2382$)$ \\
54 & 46 & $4229(2590$ to 5754$)$ \\
60 & 40 & $5353(3278$ to 7301$)$ \\
70 & 30 & $7291(4423$ to 10025$)$ \\
100 & 0 & $13639(7838$ to 19308$)$
\end{tabular}

*Refers to breast feeding or no breast feeding for up to 3 months.

UK in 2002. We have estimated the number of cases of coeliac disease, asthma and obesity that could be prevented in this population over 7, 8 and 9 years, respectively, if all the babies had been breast fed. We have also estimated the number of cases that could be prevented at different prevalence levels of breast feeding. The results of this study show that the impact of low rates of breast feeding on the UK population with regard to these chronic disorders is significant. Increasing the prevalence of breast feeding could help reduce the incidence of these disorders.

The use of the PIN-ER- $t$ statistic allows the population impact of "lack of breast feeding" to be expressed in simple numbers which could be helpful in risk communication, and would be useful in public health campaigns aimed at promoting breast feeding. PIN-ER- $t$ can be applied to other conditions which are known to be associated with lack of breast feeding in different populations in the world in order to quantify the local burden of low rates of breast feeding.

In this paper, we have assumed that the reported OR for the association between "no breast feeding" and asthma, coeliac disease and obesity approximated to RR. When the prevalence of the outcome of interest is small, the OR will approximate to the RR, but it must be noted that ORs generally tend to overestimate the size of the effect compared with RRs. ${ }^{5}{ }^{15}$

It must also be noted that the estimated population impact numbers above (33 100 for asthma, 2655 for coeliac disease and 13639 for obesity) are only achievable if "no breast feeding" as a risk factor at the specified times was completely eliminated in babies in England and Wales born in 2002, that is if all the babies were breast fed. However, such an aspiration will not be realistic as the chance of getting all mothers to breast feed their babies for months is very low indeed. It is however possible to estimate cases of the disorders that could be prevented if the current prevalence of breast feeding was increased to higher prevalence levels. Tables 1-3, respectively, show the number of cases of asthma, coeliac disease and obesity that could be prevented at different prevalence levels of breast feeding. For example, for asthma, the number of cases that could be prevented in this population over 9 years are 3759, 9475, 12104 and 16753 at breast feeding prevalence rates of $40 \%, 54 \%, 60 \%$ and $70 \%$, respectively.

\section{CONCLUSION}

The population burden of low breast feeding rates is high with regard to these chronic disorders. Improving breast feeding rates could lead to significant reductions in the incidence of these disorders. The use of PIN-ER- $t$ allows the population impact of low breast feeding rates to be quantified and communicated in a way that will make it easier for both the general public and decision makers to understand.

\section{What is already known on this topic}

- Lack of breast-feeding has been reported to be associated with a number of chronic childhood disorders.

- A recently described statistic, the "population impact number of eliminating a risk factor over a time period $(P I N-E R-t)^{\prime \prime}$ allows the impact of risk factors for disease in a population to be quantified and communicated in a way that is easier to understand.

\section{What this study adds}

- The population burden of low breast-feeding rates in the UK is high with regard to asthma, coeliac disease and obesity.

- Improving breast-feeding rates could lead to significant reductions in the incidence of these disorders. In the population of the 596122 babies born in England and Wales in 2002, about 33100 cases of asthma, 2655 cases of coeliac disease and 13639 cases of obesity could be prevented over 7-9 years if "no breastfeeding" as a risk factor was eliminated.

\section{Authors' affiliations}

Anthony K Akobeng, Department of Paediatric Gastroenterology, Central Manchester and Manchester Children's University Hospitals, Booth Hall Children's Hospital, Manchester, UK

Richard F Heller, Evidence for Population Health Unit, Division of Epidemiology and Health Sciences, University of Manchester, Manchester, UK

Competing interests: None declared.

\section{REFERENCES}

1 Leung AK, Sauve RS. Breast is best for babies. J Natl Med Assoc 2005:97:1010-9.

2 World Health Organization. Governments must promote breastfeeding, says WHO and UNICEF. Bull World Health Organ 2004;82:318.

3 Davis MK. Breastfeeding and chronic disease in childhood and adolescence. Pediatr Clin North Am 2001;48:125-41.

4 Heller RF, Buchan I, Edwards R, et al. Communicating risks at the population level: application of population impact numbers. BMJ 2005;327:1 162-5.

5 Davies HT, Crombie IK, Tavakoli M. When can odds ratios mislead? BMJ 1998;316:989-91

6 Office of National Statistics. Birth statistics - review of the Registrar General on birth and patterns of family building in England and Wales, 2002. HMSO: London, 2004.

7 Hamlyn H, Brooker S, Oleinikova K, et al. Infant feeding 2000. London: Stationery Office, 2002.

8 Gdalevich M, Mimouni D, Mimouni M. Breast-feeding and the risk of bronchial asthma in childhood: a systematic review with meta-analysis of prospective studies. J Pediatr 2001;139:261-6.

9 McCann D, McWhirter J, Coleman H, et al. The prevalence and management of asthma in primary-aged school children in the south of England. Health Educ Res 2002; 17:181-94

10 Akobeng AK, Ramanan AV, Buchan I, et al. Effect of breast feeding on risk of coeliac disease: a systematic review and meta-analysis of observational studies. Arch Dis Child 2006;91:39-43.

11 Bingley PJ, Williams AJ, Norcross AJ, et al. Avon Longitudinal Study of Parents and Children Study Team. Undiagnosed coeliac disease at age seven: population based prospective birth cohort study, BMJ 2004;328:322-3.

12 Harder T, Bergmann R, Kallischnigg G, et al. Duration of breastfeeding and risk of overweight: a meta-analysis. Am J Epidemiol 2005;162:397-403.

13 Jotanglia D, Moody A, Stamakakis E, et al. Health survey for England: obesity among children under 11. London: Stationery Office, 2005

14 Brownson RC, Baker EA, Leet TL, et al. Evidence based public health. New York: Oxford University Press, 2003.

15 Zhang J, Yu KF. What's the relative risk? A method of correcting the odds ratio in cohort studies of common outcomes. JAMA 1998;280:1690-1. 